This is a post-peer-review, pre-copyedit version of an article published in

J Mech Behav Biomed Mater . 2020 Feb;102:103459. doi: 10.1016/j.jmbbm.2019.103459.

1

2

3

4

5

6

7

8

9

\title{
Biorelevant polyanions stabilize fibrin against mechanical and proteolytic decomposition: effects of polymer size and electric charge
}

\author{
Erzsébet Komorowicz', Nóra Balázs', Anna Tanka-Salamon', Zoltán Varga², László \\ Szabó $^{1}$, Attila Bóta ${ }^{2}$, Colin Longstaff ${ }^{3}$, Krasimir Kolev ${ }^{1^{*}}$ \\ ${ }^{1}$ Department of Medical Biochemistry, Semmelweis University, Budapest, Hungary \\ ${ }^{2}$ Department of Biological Nanochemsitry, Institute of Materials and Environmental Chemistry, \\ Research Centre for Natural Sciences, Hungarian Academy of Sciences, Budapest, Hungary \\ ${ }^{3}$ Biotherapeutics, Haemostasis Section, National Institute for Biological Standards and \\ Control, South Mimms, Potters Bar, UK \\ *Correspondence:
}

Krasimir Kolev, Semmelweis University, Department of Medical Biochemistry, 1094 Budapest, Tủzoltó utca 37-47., Hungary, tel.: +36 14591500/60035, fax: +36 12670031 , e-mail: Krasimir.Kolev@eok.sote.hu

Keywords: Fibrin, Fibrinolysis, Heparin, Polyphosphate, Plasmin

\begin{abstract}
Abbreviations: LMWH, low molecular-weight heparin; NETs, neutrophil extracellular traps; S5, pentasaccharide; SEM, scanning electron microscopy; tPA, tissue-type plasminogen activator; UFH, unfractionated heparin
\end{abstract}




\begin{abstract}
The release of neutrophil extracellular traps (NETs) containing DNA and histones is an essential mechanism in the neutrophil-mediated innate immunity.-In thrombi the polyanionic DNA confers mechanical and lytic resistance to fibrin and heparins interfere with the effects of NET components. Heparins are polyanions used not only as therapeutic agents, but they are also released by mast cells at entry sites of pathogens. Platelets and microorganisms release a different type of polyanions (polyphosphates) of various size (in the range 60-1000 phosphate monomers). With the current study we aimed to evaluate if the stability of fibrin is influenced by the type of polyanion, its molecular size or relative electric charge.
\end{abstract}

Fibrin structure was approached with scanning electron microscopy (SEM) and pressure-driven permeation. An oscillation rheometer was used to investigate viscoelastic properties. Kinetic turbidimetric assays for the generation and dissolution of composite fibrin clots containing unfractionated heparin (UFH), and its partially or fully desulfated derivatives, as well as low molecular-weight heparin (LMWH), pentasaccharide (S5), and polyphosphates composed of 45 (P45), 100 (P100) or 700 (P700) monomers at average.

The smaller polyanions P45, P100, LMWH, and S5 accelerated, whereas P700 and UFH retarded clot formation. All polyanions altered the fibrin structure: SEM and clot permeation showed thicker fibers with smaller (LMWH, S5, P700) or larger (UFH, P100) pores. All polyanions stabilized the clots mechanically, but the smaller P45, P100 and LMWH decreased the deformability of fibrin, whereas the large UFH and P700 increased the maximal bearable deformation of clots. Despite the size-dependent structural changes, all heparins caused a 10$15 \%$ prolongation of lysis-times with plasmin, and UFH-effects depended on sulfation patterns. The 20-35\% prolongation of lysis-times caused by all polyphosphates was a kringle-dependent phenomenon, and was dampened in the presence of 6-aminohexanoate blocking the lysinebinding sites of plasmin.

In summary, we found that polyanions of different chemical structure stabilize fibrin clots via size-dependent modulation of fibrin structure and kringle-dependent inhibition of plasminmediated fibrinolysis. 


\section{Heparins and polyphosphates in fibrin}

\section{Introduction}

Fibrin structure provides a solid scaffold in hemostatic and thrombotic clots, functions as the primary matrix in the wound healing process, and is also applied as a therapeutic biomaterial (Mossesson, 2005; Laurens et al., 2006; Brown and Barker, 2014). Fibrin is also generated at sites of inflammation, where neutrophils incorporated into fibrin can release neutrophil extracellular traps (NETs) containing DNA (a polyanion) and histones. Multiple interactions have been discovered between NET components and the hemostatic-fibrinolytic system, including DNA-mediated changes in fibrin structure and size-dependent inhibitory effects of DNA on fibrinolysis (Martinod and Wagner, 2014; Varju et al., 2015; Engelmann and Massberg, 2013). Formation of fibrin at sites of microbial infection could be both an advantage and a disadvantage to the host (Loof et al., 2014; Mullarky et al., 2005).

Thrombin, a serine-protease activated in the blood coagulation cascade cleaves plasma-soluble fibrinogen into fibrin monomers, which polymerize into protofibrils, and form a three-dimensional network structure via further lateral aggregation of protofibrils and branching of fibrin fibers (Weisel and Litvinov, 2013). Fibrin fiber thickness, branching density and porosity of patients' plasma clots have been linked to the frequency of cardiovascular events and thrombolytic resistance (Undas and Ariens, 2011). The lytic susceptibility of extravascular fibrin may influence the spreading of pathogens, as well as the wound healing process (Laurens et al., 2006; Loof et al., 2014).

Previously we have described the differential modulation of fibrin network structure and stability by DNA in pure fibrin (Longstaff et al., 2013) or plasma clots (Varju et al., 2015), as well as by several glucosaminoglycans including chondroitin sulfate, dermatan sulfate, and hyaluronic acid (Rottenberger et al., 2013; Komorowicz et al., 2017). These fibrin-modifiers with variable anionic charge may influence cell proliferation and migration, wound healing and tumor development when incorporated into arterial thrombi over atherosclerotic lesions or into inflammation-related extravascular fibrin structures. Mechanical, as well as fibrinolytic resistance of composite fibrin networks have also been an important point of consideration for the application of fibrin in tissue engineering and drug delivery (Janmey et al., 2009).

Heparins are anionic, sulfated glycosaminoglycan polymers that have long been administered as an anticoagulant for the prevention and therapy of thrombosis, since they accelerate the inactivation of thrombin and factor Xa by antithrombin. Unfractionated heparin (UFH) is heterogeneous with respect to its size with molecular weight in the 3,000-30,000 Da range, averaging at $15,000 \mathrm{Da}$ (equal to approximately 50 monosaccharide units). The lowmolecular weight heparins (LMWHs), containing a mixture of shorter polysaccharide chains with molecular weights in the 2,000-10,000 Da range, averaging at 5,000 Da), whereas a synthetic pentasaccharide (S5), fondaparinux, represents the minimal sequence motif of heparin, which can still bind to antithrombin (Paolucci, 2002).

The hemostatic effects of heparin and its derivatives are not restricted to their anticoagulant action, but they can also modify the structure of the fibrin matrix. Therapeutic concentrations of S5 $(0.5 \mathrm{mg} / \mathrm{l})$ and UFH (up to $1 \mathrm{U} / \mathrm{ml})$ resulted in thicker fibrin fibers and more porous, less branched fibrin network enabling better penetration of tissue-type plasminogen activator (tPA), and hence faster proteolytic disassembly of fibrin in plasma clots in vitro (Nenci et al., 1992; Varin et al., 2007). However, other data questioned the effects of UFH on fibrinolysis, and depending on the experimental conditions controversial data with LMWHs have also been reported (Parise et al., 1993; Weitz et al, 1991). The sulfation pattern of the heparin glycosaminoglycan chain appears to play a critical role not only in its 
anticoagulant activity, but also in its interaction with a number of physiological ligands, such as growth factors, cell adhesion receptors, cytokines, and histones (Longstaff et al., 2016; Fryer et al., 1997; Jouni et al., 2016; Wan et al., 2002).

Besides glycosaminoglycans, polyphosphates, the inorganic polymers of orthophosphates connected by phosphoanhydride bonds, represent another group of polyanions, which were earlier best known for their role in energy homeostasis. Platelets store relatively short polyphosphate chains (60-100 phosphate units) in their dense granules (at concentrations up to $130 \mathrm{mM}$ ), and release them upon platelet activation and granule secretion (Morrissey et al., 2012; Ruiz et al., 2004). Polyphosphates of microbial origin are much longer (200-1,000 phosphate units), and several prothrombotic actions of polyphosphates have been described to be dependent on the chain-length of the polyphosphate (Morrissey et al., 2012), but their sizedependent effects on fibrin network structure and stability remained largely uncharacterized.

Our present work addresses the size-, and sulfation-dependent modulation of fibrin structure and stability by heparins and polyphosphates in purified, in vitro experimental systems with the ultimate goal to improve the understanding of the direct impact of these biologically relevant polyanions on the function of fibrin as a biomechanical barrier in inflammatory loci or scaffold of vascular clots. 


\section{Heparins and polyphosphates in fibrin}

\section{Materials and Methods}

Human fibrinogen (plasminogen free) was the product of Calbiochem (LaJolla, CA, USA). The chromogenic substrates for plasmin Spectrozyme-PL (H-D-norleucylhexahydrotyrosyl-lysine-p-nitroanilide), and for thrombin Spectrozyme-TH (H-Dhexahydrotyrosyl-L-alanyl-L-arginine-p-nitroanilide) were from Sekisui Diagnostics (Pfungstadt, Germany). Bovine thrombin purchased from Serva (Heidelberg, Germany) was further purified by ion-exchange chromatography on sulfopropyl-Sephadex yielding preparation with specific activity of 2,100 IU/mg (Lundblad et al., 1976) and $1 \mathrm{IU} / \mathrm{ml}$ was considered equivalent to approximately $10.7 \mathrm{nM}$ by active site titration (Longstaff, 2018). Plasminogen and plasmin were prepared as previously described (Varju, 2015).

Heparins used were unfractionated heparin (UFH, Heparin Sodium, Wockhardt, Wrexham, UK) and low molecular weight heparin (LMWH, WHO, International Standard code 11/176, 342 anti-thrombin IU and 1068 anti-factor Xa IU per ampoule; and Enoxaparin, NIBSC reagent code 11/174, 275 anti-IIa IU and 1030 anti-FXa IU per ampoule, NIBSC, S Mimms UK). Modified heparins were a partially desulfated version of $\mathrm{N}$-desulfated re-N-acetylated heparin (Nagasawa et al., 1977), and a fully desulfated variant, N-acetylated de-O-sulfated heparin, characterised by NMR. The pentasaccharide fondaparinux sodium (S5) was the product of GlaxoSmithKline, London, UK. Polyphosphate (High MW, P700) with approximate polymer lengths ranging from $\sim 200-1,300$ phosphate units (modal size about 700 phosphate units) and polyphosphate (Medium Chain, P100) with approximate polymer lengths ranging from 45-160 phosphate units (modal size about 75 phosphate units) were purchased from Kerafast, Boston, MA, USA. Short-chain polyphosphate (P45) with average polymer length 4050 phosphate units was from Sigma-Aldrich Kft. (Budapest, Hungary).

In order to establish the relative strength of the effects on fibrin structure and lysibility, all heparin derivatives were applied at concentrations relevant to their therapeutic levels and comparison was made on the basis of equal mass, because molarity is not feasible for the assessment of materials with heterogeneous molecular size. Taking into account the specific activities of the used UFH and LMWH species, we chose $2.5 \mathrm{mg} / 1$ and $10 \mathrm{mg} / \mathrm{l}$ values, and added one more concentration of $0.5 \mathrm{mg} / \mathrm{l}$, as the therapeutic concentration for the S5 (Hirsh and Fuster, 1994; Varin et al., 2007). Polyphosphates were compared on the basis of monomer phosphate concentration in the $0-400 \mu \mathrm{M}$ range, which is relevant according to in vivo estimates around platelets, and previously published observations (Morrissey et al., 2012).

\subsection{Characterization of the structure of fibrin clots}

Scanning electron microscopy (SEM) was used for the measurement of fiber thickness in the fibrin network structures. Fibrin clots were prepared in triplicates: $6 \mu \mathrm{M}$ fibrinogen in 10 mM HEPES buffer $\mathrm{pH} 7.4$ containing $150 \mathrm{mM} \mathrm{NaCl}$ (HBS) and the polyanion macromolecules at the examined concentrations was clotted with thrombin at $8 \mathrm{nM}$ for $1 \mathrm{~h}$ (when heparin and its derivatives were evaluated) or with thrombin at $5 \mathrm{nM}$ for $2 \mathrm{~h}$ (when polyphosphates were used). Clots were processed for SEM imaging as detailed previously (Rottenberger et al., 2013) and images were taken with scanning electron microscope EVO40 (Carl Zeiss GmbH, Oberkochen, Germany). SEM images were analyzed to determine the distribution of fibrin fiber diameters using self-designed program functions running under the Image Processing Toolbox v. 10.3 of Matlab R2018b (The Mathworks, Natick, MA, USA) as previously described (Longstaff et al., 2011; Nikolova et al., 2013). The diameter of 300 fibers was measured on the SEM images ( 3 independent samples for each clot type). The distribution curves of fibrin fiber 


\section{Heparins and polyphosphates in fibrin}

thickness in the clots were characterized by the median and interquartile range (IQR) of the diameter values.

Fluid permeability of the clots was measured to assess the porosity of the fibrin network. Fibrin clots were formed at the bottom of disposable plastic pipette tips using $15 \mathrm{nM}$ thrombin and $7.4 \mu \mathrm{M}$ fibrinogen in HBS, and the polyanion macromolecules at various concentrations. After 70 min of clotting at $37^{\circ} \mathrm{C}$, the rate of fluid flow through the clots was measured, while hydrostatic pressure between the top surface of the buffer reservoir and the bottom of the clot was kept constant. The permeability coefficient, $K_{s}$ was calculated, as previously published (Varju et al., 2015) and results are expressed in relative units compared to the $K_{s}$ of fibrin without any additive.

Viscoelastic properties of the fibrin clots were studied with oscillation rheometry (Komorowicz et al., 2017). Fibrinogen (7.4 $\mu \mathrm{M}$ in HBS) was pre-mixed with the heparin or polyphosphate variants, and after the addition of $10 \mathrm{nM}$ thrombin the clotting mixture was immediately transferred to the stationary plate of HAAKE RheoStress 1 oscillation rheometer (Thermo Scientific, Karlsruhe, Germany). The cone (Titanium, $2^{\circ}$ angle, $35 \mathrm{~mm}$ diameter, 105 $\mu \mathrm{m}$ default gap size) of the rheometer was brought to the gap position and an oscillatory shear strain $(\gamma)$ of 0.015 at $1 \mathrm{~Hz}$ was imposed at $2 \mathrm{~min}$ after the addition of thrombin. Measurements of storage modulus $\left(G^{\prime}\right)$ and loss modulus $\left(G^{\prime \prime}\right)$ were taken for 10 min with HAAKE RheoWin data manager software v. 3.50.0012 (Thermo Scientific). Following this 10-min clotting phase, determination of the flow limit of the fibrin gels was performed in the same samples increasing the applied shear stress $(\tau)$ from 0.01 to $1000 \mathrm{~Pa}$ stepwise in $300 \mathrm{~s}$ and the resulting strain was measured and used for calculation of the viscosity modulus $(\eta)$. The gel-fluid transition in the composite fibrin structure was indicated by an apparent fall in viscosity to zero, which point could be characterized by two parameters: the maximal bearable strain $\left(\gamma_{\max }\right)$ preceding the abrupt fall in viscosity and the critical shear stress $\tau_{0}$ that resulted in the $\gamma_{\max }$. Since there is always a theoretical possibility of sample detachment from the cone-head or the slipping of sample on the stationary plate, we have addressed the hypothesis for a potential wall slip phenomenon with the measurements presented as Supplementary Material, and based on these we concluded that it is highly unlikely that a wall slip would occur using the instrumental setup and perturbation procedures used in our study.

\subsection{Turbidimetric fibrinolytic assays}

Formation and dissolution of fibrin clots were followed in 96-well microtiter plates by measuring the light absorbance at $340 \mathrm{~nm}$ at $37^{\circ} \mathrm{C}$ with a Zenyth 200rt microplate spectrophotometer (Anthos Labtec Instruments GmbH, Salzburg, Austria).

Intrinsic fibrinolysis was initiated by mixing $6 \mathrm{nM}$ plasmin in the clot at the time of the initiation of clotting by thrombin. Fibrinogen $(6 \mu \mathrm{M})$ in HBS containing $0.5-10 \mathrm{mg} / \mathrm{l}$ heparin variants or $5-400 \mu \mathrm{M}$ polyphosphates was mixed with $20 \mathrm{nM}$ thrombin and $6 \mathrm{nM}$ plasmin in the well. In these experimental setups fibrin formation and dissolution are concurring processes, so when picking enzyme concentrations care was taken to reach maximal clot turbidities $\left(A_{\max }\right)$ within a $10 \%$ difference from $\mathrm{A}_{\max }$ attained in the absence of plasmin.

In our extrinsic fibrinolysis model dissolution of preformed clots was initiated by applying plasmin on the clot surface. Fibrinogen at $6 \mu \mathrm{M}$ in HBS containing $0.5-10 \mathrm{mg} / \mathrm{l}$ heparin variants or 5-400 $\mu \mathrm{M}$ polyphosphates was clotted with 5-20 $\mathrm{nM}$ thrombin in the wells and fibrin formation was monitored by turbidimetry at $340 \mathrm{~nm}$ wavelength $\left(\mathrm{A}_{340}\right)$. After the clot turbidity reached a plateau $\left(\mathrm{A}_{\max }\right)$, indicating the end of the first phase, fibrinolysis was initiated by 
layering $90 \mu 1500 \mathrm{nM}$ plasmin on the surface of the clots and the turbidimetric measurement was continued to detect the dissolution process. Due to differential modulation of $\mathrm{CT}_{90}$ by the various additives measured concurrently in the same microplate, the first phase of extrinsic lysis curves varied in the range of 40-100 min. The role of the high-affinity lysine-binding sites located on the kringle ${ }_{1-3}$ domains was studied by including the blocking lysine-analogue 6aminohexanoate at $0.5 \mathrm{mM}$ concentration in the plasmin solution.

For quantitation of the turbidimetric experiments clotting time $\left(C T_{90}\right)$ was defined as the time needed to reach $90 \%$ of the maximal turbidity, $\mathrm{A}_{\max }$, on the ascending part of the turbidimetric curve., whereas Lysis time $\left(L T_{50}\right)$ was defined as the time needed to reduce the turbidity of the clot to a half-maximal value in the course of its dissolution. In the extrinsic fibrinolytic setup $L T_{50}$ was measured from the time of plasmin addition.

\subsection{Statistical analysis}

The distribution of the data on fiber diameter was analyzed according to an algorithm used previously (Longstaff et al., 2011; Nikolova et al., 2013). The differences between pairs of fitted 1-D distributions of fiber diameters were assessed for statistical significance based on Kuiper's test. A Monte Carlo simulation procedure was used to construct the Kuiper statistic's distribution, for which the previously described platform with original program functions running under Matlab R2018b was applied (Nikolova et al., 2013). The statistical evaluation of the rest of the experimental measurements in this report was performed with the two-sample nonparametric Kolmogorov-Smirnov test that evaluates the difference between mean values of datasets without any assumptions about the character of their distribution functions (Statistics and Machine Learning Toolbox v. 11.4 of Matlab R2018b, The MathWorks Inc., Natick, MA, USA).

\section{Results}

\subsection{Effects of heparin derivatives on fibrin clot formation and structure}

Among the heparins, both the LMWH and the S5 accelerated fibrin formation (shorter $\mathrm{CT}_{90}$ ) resulting in a final network with higher turbidity (Table 1 ). The median fiber diameter increased and the distribution of the diameter values showed a narrower spread (smaller IQR), indicating a more homogeneous fibrin structure. These thicker fibers encircled smaller pores reflected in the lower permeability constant. The largest, unfractionated heparin (UFH) retarded clot assembly even in the absence of antithrombin, and a more porous network was formed containing thicker and more polydisperse fibers (Table 1). These effects of UFH were mostly sulfation-dependent, although even the fully desulfated UFH displayed a partial fiberthickening effect (Table 1). 
Table 1 Kinetic and structural parameters of fibrin formation in the presence of heparins of various size and degree of sulfation.

\begin{tabular}{|c|c|c|c|c|}
\hline Additive & $\mathrm{CT}_{90}(\mathrm{RU})$ & $A_{\max }(R U)$ & $D(\mathrm{~nm})$ & $K_{s}(\mathrm{RU})$ \\
\hline none & $1.00(0.14)$ & $1.00(0.12)$ & $54.0[23.2]$ & $1.00(0.04)$ \\
\hline LMWH & $0.75 *(0.10)$ & $1.26 *(0.09)$ & $56.9 *[18.8]$ & $0.51 *(0.19)$ \\
\hline \multicolumn{5}{|l|}{ Pentasaccharide } \\
\hline $0.5 \mathrm{mg} / \mathrm{L}$ & $0.89(0.10)$ & $1.18 *(0.06)$ & $59.1 *[18.2]$ & $0.90 *(0.09)$ \\
\hline $2.5 \mathrm{mg} / \mathrm{L}$ & $0.50 *(0.08)$ & $1.18 *(0.05)$ & $63.4 *[17.6]$ & $0.72 *(0.06)$ \\
\hline UFH & $1.50 *(0.23)$ & $1.06(0.10)$ & $67.3 *[29.2]$ & $1.47 *(0.06)$ \\
\hline UFH-Ndes & $0.81 *(0.22)$ & $1.09 *(0.06)$ & $70.0 *[30.4]$ & $0.73 *(0.08)$ \\
\hline UFH-Des & $0.89(0.25)$ & $0.99(0.06)$ & $62.0 *[25.4]$ & $1.05(0.07)$ \\
\hline
\end{tabular}

Fibrin clots containing LMWH, pentasaccharide fondaparinenux, or parent, partially desulfated and fully desulfated heparin variants (UFH, UFH-Ndes, and UFH-Des, respectively) at $2.5 \mathrm{mg} / \mathrm{l}$ (if not indicated otherwise) were prepared as detailed in Materials and Methods. The kinetics of clot formation was characterized in turbidimetric experiments by the clotting time $\left(\mathrm{CT}_{90}\right)$ needed to reach $90 \%$ of maximal turbidity $\left(\mathrm{A}_{\max }\right)$ presented as mean (standard deviation) of $n=4$ experiments in relative units compared to pure fibrin. Fibrin architecture was characterized by fluid permeability coefficient, $K_{s}$ shown as mean (standard deviation) of $\mathrm{n}=12$ experiments in relative units ( $\mathrm{RU}=1$ for the permeability coefficient, $K_{s}$ of pure fibrin), as well as the fiber diameter $(D)$ from SEM images presented as median [IQR]. Asterisks indicate $p<0.05$ according to Kolmogorov-Smirnov test for comparisons to pure fibrin. The Monte Carlo Kuiper's test indicated significant differences $(p<0.001)$ between the distributions of fiber diameter of any composite clot and pure fibrin.

\subsection{Effects of polyphosphates on fibrin clot formation and structure}

The effects of polyphosphates were also size-dependent, the shorter P45 and P100 accelerated fibrin formation and slightly decreased clot turbidity, whereas the large P700 caused the opposite changes in the kinetics of fibrin formation and final turbidity (Table 2). All polyphosphates resulted in thicker fibers (the thickest fibers were found with the largest P700), and the longer variants widened the probability distribution functions of the diameter values indicating increased heterogeneity of the fibrin meshwork. In contrast to the size-trend of the heparin effects on fibrin permeability, the fibrin clots containing shorter polyphosphate were more porous than the clots with P700.

Table 2 Kinetic and structural parameters of fibrin formation in the presence of polyphosphates of various size.

\begin{tabular}{lllll}
\hline Additive & $\mathrm{CT}_{90}(\mathrm{RU})$ & $\mathrm{A}_{\max }(\mathrm{RU})$ & $D(\mathrm{~nm})$ & $K_{s}(\mathrm{RU})$ \\
\hline none & $1.00(0.12)$ & $1.00(0.05)$ & $68.0[23.1]$ & $1.00(0.05)$ \\
$\mathrm{P} 45$ & $\mathbf{0 . 5 9 *}(\mathbf{0 . 2 5})$ & $\mathbf{0 . 8 5} * \mathbf{( 0 . 0 7 )}$ & $\mathbf{7 2 . 7} *[\mathbf{2 4 . 1}]$ & $0.87(0.11)$ \\
$\mathrm{P} 100$ & $\mathbf{0 . 3 8 * ( \mathbf { 0 . 0 6 } )}$ & $\mathbf{0 . 8 1} * \mathbf{( 0 . 0 6 )}$ & $\mathbf{7 2 . 4 * [ 3 1 . 3}$ & $\mathbf{1 . 5 1 *}(0.07)$ \\
$\mathrm{P} 700$ & $\mathbf{1 . 2 0 * ( \mathbf { 0 . 2 9 } )}$ & $\mathbf{1 . 2 2} * \mathbf{( 0 . 1 0 )}$ & $\mathbf{7 3 . 6 * [ 3 4 . 5}$ & $\mathbf{0 . 5 9 *}(0.22)$ \\
\hline
\end{tabular}

Fibrin clots containing polyphosphates of various length at $400 \mu \mathrm{M}$ monomeric phosphate concentration were prepared as detailed in Materials and Methods. The reported parameters (clotting time, $\mathrm{CT}_{90}$; maximal turbidity, $\mathrm{A}_{\max }$; fiber diameter, $D$; permeability coefficient, $K_{s}$ ) were measured and defined as described in Table 1 . Asterisks indicate $p<0.05$ according to Kolmogorov-Smirnov test for comparisons to pure fibrin. The Monte Carlo Kuiper's 
test indicated significant differences $(p<0.001)$ between the distributions of fiber diameter of any composite clot and pure fibrin.

\subsection{Viscoelasticity of fibrin containing polyanions}

The viscoelastic properties of the composite clots were studied in two experimental setups. At first, the development of clot strength in the course of fibrin formation was measured under oscillatory shear strain, and the plateau values of storage and loss moduli, as well as their ratio, the loss tangent were computed (Fig. 1). In the second stage, the fibrin clot was exposed to a stepwise increasing shear stress until structural disintegration or gel-fluid transition occurred and the resulting strain was measured and used for calculation of the gel viscosity. The plots of viscosity versus shear stress generated flow curves that were used for the determination of the maximal bearable deformation, and the critical shear stress at the gel-fluid transition point (Fig. 2). Heparins and polyphosphates modified the development of clot strength in the course of fibrin formation in a sulfation-, and size-dependent manner. The S5 and the fully desulfated UFH-derivative did not alter the viscoelastic parameters (Tables 4 and 5). The rate of clot strengthening in the course of fibrin polymerization, however, was changed even by S5: the shorter polyanions P45, P100, as well as LMWH and S5 all accelerated clot strengthening, whereas the two longest polymers - polyphosphate P700 and UFH - retarded it (Figs. 1 and 3). Typically the storage moduli characterizing the elastic deformability were elevated by all polyanions except for S5 and P700, suggesting the formation of stronger structures (Tables 3-5). The loss moduli describe the viscous, energy-loss component of the deformation, and drops in the loss tangent, as observed with LMWH and the shorter polyphosphates, suggest relatively less energy loss, less structural alteration in the course of the deformation (Tables 3 and 5). The composite clots with higher storage moduli were less easily deformable under stepwise increasing shear stress (dynamic viscosity panels in Figs. 2 and 4) and required typically a higher critical shear stress for structural failure. The magnitude of relative deformation at various applied shear stress (panels B in Figs. 2,4) revealed some more details on the background of critical shear stress changes. Clots containing the large P700 and UFH disintegrated at a significantly higher degree of deformation than pure fibrin, whereas clots containing the two shorter polyphosphates, LMWH or S5 were strong, but stiff, since they allowed for much less deformation than pure fibrin clots (Figs. 2,4 and Tables 3-5).

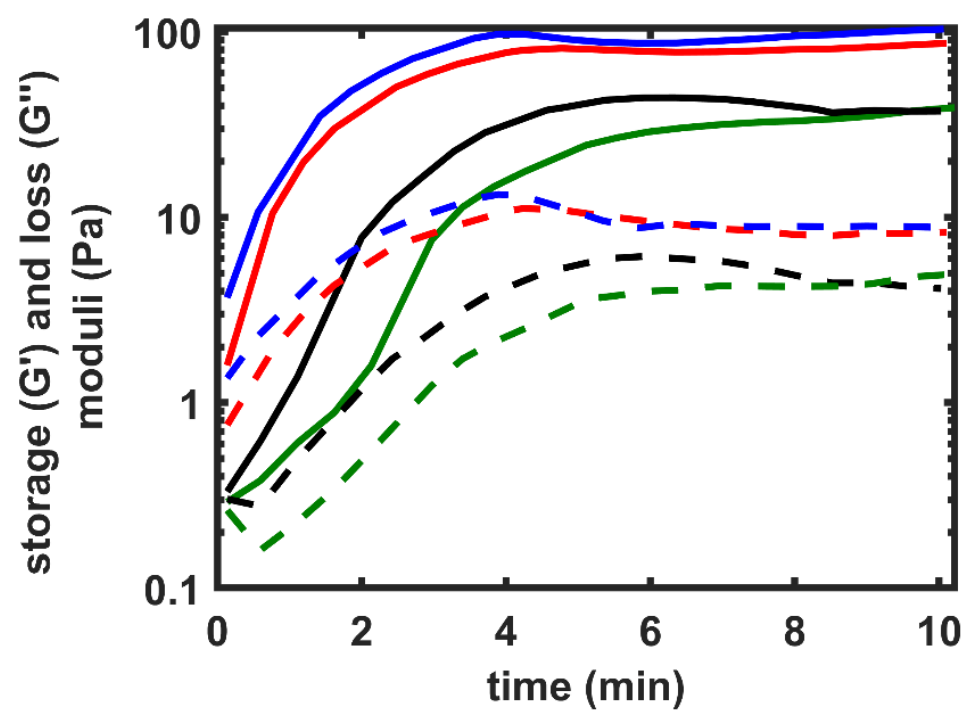

Fig. 1 Kinetics of viscoelasticity changes in the course of fibrin formation in the presence of polyphosphates. Fibrinogen (7.4 $\mu \mathrm{M})$ containing no additive (black) or polyphosphates of 45 (red), 100 (blue) or 700 (green) units (all at $400 \mu \mathrm{M}$ monomer concentration) was mixed with $10 \mathrm{nM}$ thrombin and storage modulus ( $\mathrm{G}^{\prime}$, continuous line), as well as loss modulus $\left(\mathrm{G}^{\prime}\right.$, dashed line) were measured in an oscillation rheometer in the course of time. Numerical data for the maximal G' and G', values are summarized in Table 3. 


\begin{tabular}{|c|c|c|c|c|}
\hline & \multicolumn{4}{|c|}{ Additive } \\
\hline & none & P45 & P100 & P700 \\
\hline$G^{\prime}(\mathrm{Pa})$ & $39.45(2.08)$ & $78.81 *(12.67)$ & $100.61 *(3.09)$ & $37.29(3.75)$ \\
\hline $\mathrm{G}^{\prime \prime}(\mathrm{Pa})$ & $4.72(0.51)$ & $7.68 *(0.54)$ & $8.75 *(0.43)$ & $4.81(0.45)$ \\
\hline$G^{\prime} ' / G^{\prime}(-)$ & $0.119(0.007)$ & $0.099 *(0.015)$ & $0.086 *(0.002)$ & $0.129(0.002)$ \\
\hline$\tau_{0}(\mathrm{~Pa} . \mathrm{s})$ & $93.22(12.65)$ & $192.82 *(18.84)$ & $181.6 *(8.75)$ & $181.6 *(11.10)$ \\
\hline$\gamma_{\max }(-)$ & $2.26(0.11)$ & $2.69(0.50)$ & $1.81 *(0.13)$ & $5.35 *(0.17)$ \\
\hline
\end{tabular}

Fibrin clots containing various polyphosphate size-variants (P45, P100, P700; composed of 45, 100 and 700 phosphate units at $400 \mu \mathrm{M}$ ) were examined as illustrated in Figs. 1 and 2. The plateau values of the storage modulus $\left(G^{\prime}\right)$, loss modulus $\left(G^{\prime \prime}\right)$ and loss tangent $\left(G^{\prime}, / G^{\prime}\right)$ at the end of the clotting phase and the critical shear stress $\left(\tau_{0}\right)$ at the maximal bearable relative deformation $\left(\gamma_{\max }\right)$ before the gel/fluid transition in the fibrin structure are presented as mean (standard deviation). Asterisks indicate $p<0.05$ according to Kolmogorov-Smirnov test in comparison to pure fibrin, $n=4-6$.
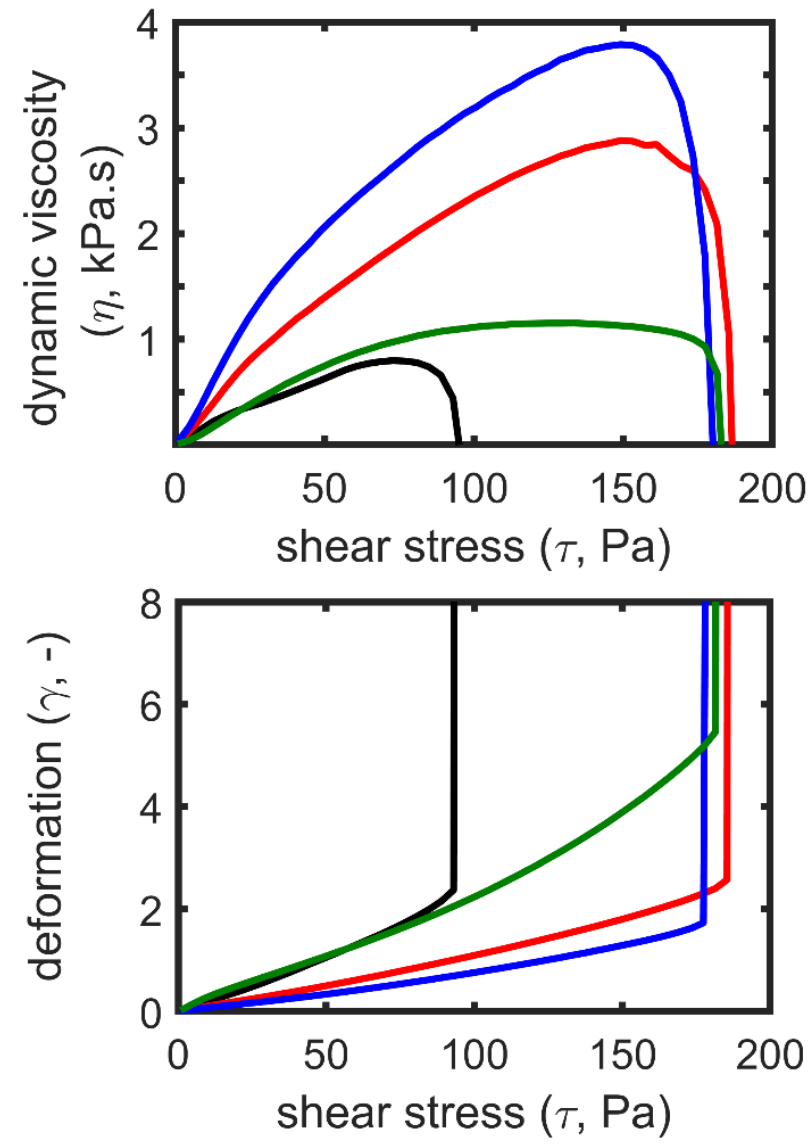

Fig. 2 Modulation of fibrin clot strength by polyphosphates. After the 10-min clotting shown in Fig.1, an increasing shear stress $\tau$ was applied to the clot formed in the gap space of the rheometer and the resulting relative deformation $\gamma$ was measured. Representative curves of the deformation $\gamma$ (lower panel), as well as the corresponding computed dynamic viscosity $(\eta)$ (upper panel) are presented for the following additives: none (black), P45 (red), P100 (blue), and P700 (green). Numerical values of the maximal bearable deformation $\left(\gamma_{\max }\right)$ and the corresponding critical stress $\left(\tau_{0}\right)$, which define the gel-fluid transition of the various clots are summarized in Table 3. 


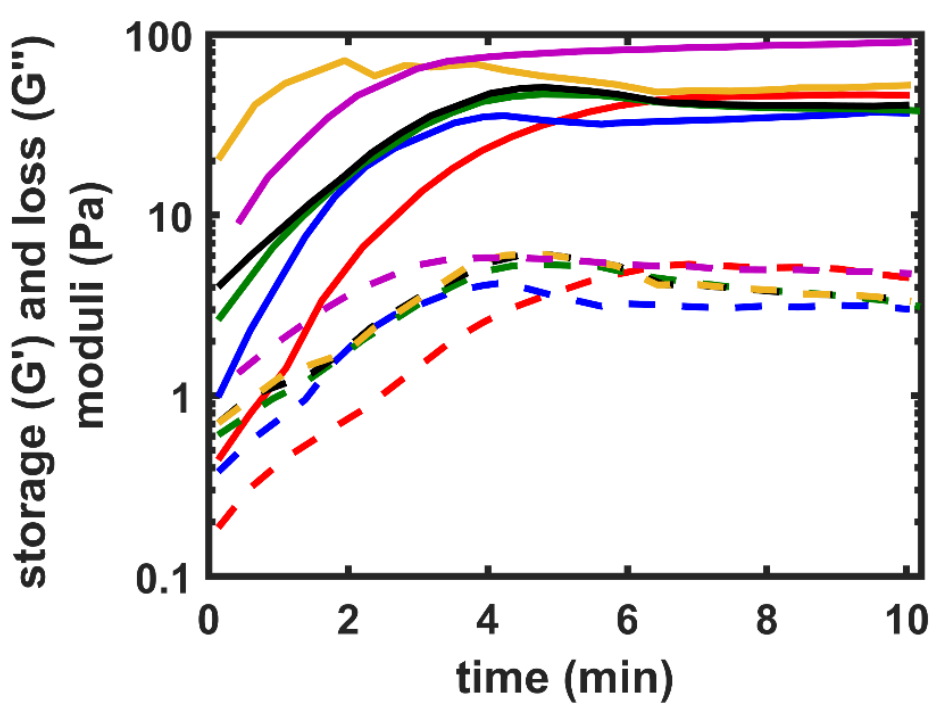

Fig. 3 Kinetics of viscoelasticity changes in the course of fibrin formation in the presence of heparin derivatives. Fibrinogen (7.4 $\mu \mathrm{M})$ containing no additive (black) or $2.5 \mathrm{mg} / \mathrm{l}$ heparin variant (parent [red], partially [blue] or fully [green] desulfated unfractionated heparin, LMWH [purple], pentasacharide [yellow]) was mixed with $10 \mathrm{nM}$ thrombin and storage modulus (G', continuous line), as well as loss modulus (G', dashed line) were measured in an oscillation rheometer in the course of time. Numerical data for the maximal G' and G" values are summarized in Tables 4 and 5.
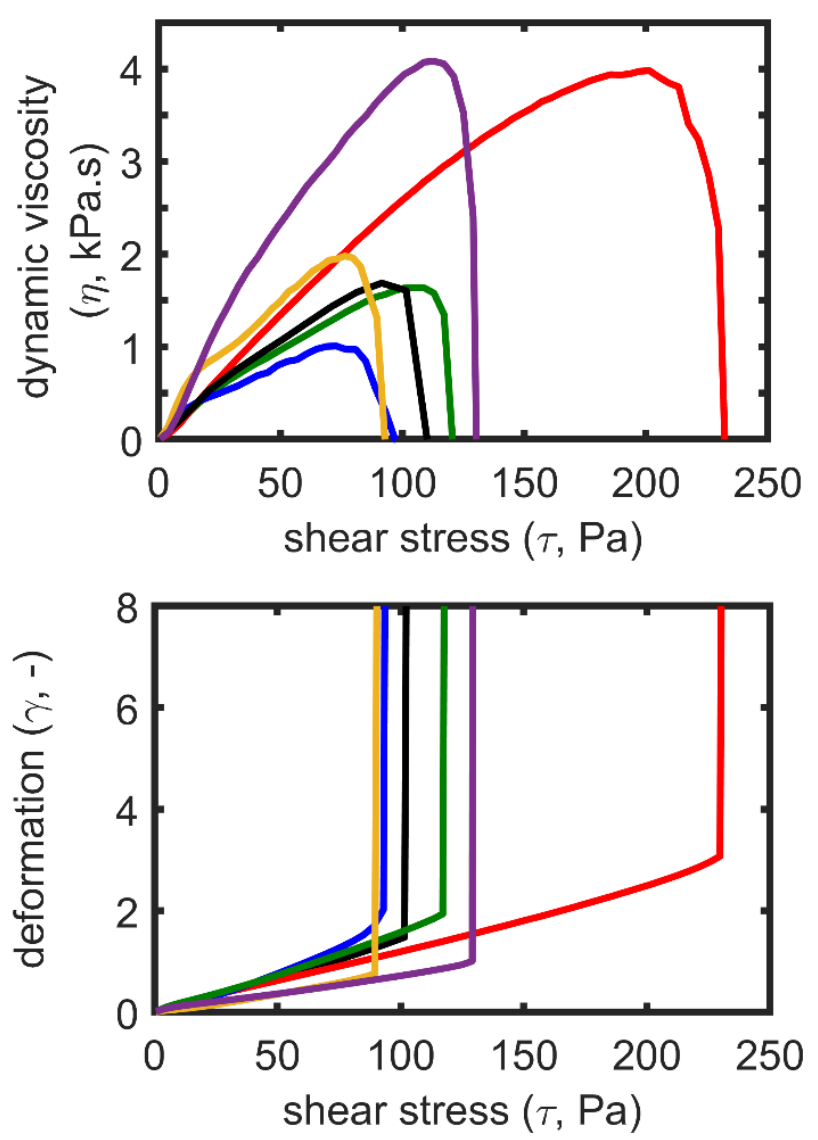

Fig. 4 Modulation of fibrin clot strength by heparins. After the 10-min clotting depicted in Fig. 3, a stepwise increasing shear stress $\tau$ was applied to the clot formed in the gap space of the rheometer and the resulting relative deformation $\gamma$ was measured. Representative curves of the deformation $\gamma$ (lower panel), as well as the corresponding computed dynamic viscosity $(\eta)$ (upper panel) are presented for the following additives: none (black); parent (red), partially (blue), or fully (green) desulfated unfractionated heparin; LMWH (purple), or pentasacharide (yellow). Numerical values of the maximal bearable deformation $\left(\gamma_{\max }\right)$ and corresponding critical stress $\left(\tau_{0}\right)$, which define the gel/fluid transition of the various clots are summarized in Tables 4 and 5 . 
Table 4. Viscoelastic parameters of composite fibrin/UFH-variant clots.

\begin{tabular}{|c|c|c|c|c|}
\hline & \multicolumn{4}{|c|}{ Additive } \\
\hline & none & UFH & UFH-Ndes & UFH-Des \\
\hline $\mathrm{G}^{\prime}(\mathrm{Pa})$ & 37.35 (3.94) & $44.98 *(1.03)$ & $25.58 *(2.94)$ & $37.58(3.75)$ \\
\hline $\mathrm{G}^{\prime \prime}(\mathrm{Pa})$ & $3.07(0.23)$ & $4.28 *(0.16)$ & $2.16 *(0.26)$ & $3.14(0.38)$ \\
\hline$G^{\prime}, / G^{\prime}(-)$ & $0.082(0.003)$ & $0.095 *(0.002)$ & $0.084(0.002)$ & $0.083(0.002)$ \\
\hline$\tau_{0}(\mathrm{~Pa} . \mathrm{s})$ & $113.36(7.24)$ & $220.02 *(11.07)$ & $76.15 *(7.92)$ & $117.4(5.42)$ \\
\hline$\gamma_{\max }(-)$ & $1.66(0.29)$ & $2.97 *(0.12)$ & $1.54(0.10)$ & $1.92(0.08)$ \\
\hline
\end{tabular}

Fibrin clots containing parent, partially desulfated and fully desulfated UFH-variants (UFH, UFH-Ndes, and UFHDes, respectively) at $2.5 \mathrm{mg} / \mathrm{l}$ were examined as illustrated in Figs. 3 and 4 . The plateau values of the storage modulus $\left(G^{\prime}\right)$, loss modulus $\left(G^{\prime \prime}\right)$ and loss tangent $\left(\mathrm{G}^{\prime} / \mathrm{G}^{\prime}\right)$ at the end of the clotting phase and the critical shear stress $\left(\tau_{0}\right)$ at the maximal bearable relative deformation $\left(\gamma_{\max }\right)$ before the gel/fluid transition in the fibrin structure are presented as mean (standard deviation). Asterisks indicate $p<0.05$ according to Kolmogorov-Smirnov test in comparison to pure fibrin, $\mathrm{n}=4-6$.

Table 5. Viscoelastic parameters of composite fibrin/LMWH-variant clots.

\begin{tabular}{lllll}
\hline & \multicolumn{4}{c}{ Additive } \\
\cline { 2 - 5 } & none & LMWH 5 mg/1 & S5 0.5 mg/l & S5 2.5 mg/1 \\
\hline $\mathrm{G}^{\prime}(\mathrm{Pa})$ & $43.82(7.29)$ & $\mathbf{8 1 . 3 2 * ( 1 9 . 1 1 )}$ & $45.45(5.06)$ & $37.15(7.59)$ \\
$\mathrm{G}^{\prime}{ }^{\prime}(\mathrm{Pa})$ & $3.69(0.76)$ & $4.09(1.50)$ & $3.57(0.43)$ & $3.35(0.43)$ \\
$\mathrm{G}^{\prime}{ }^{\prime} \mathrm{G}^{\prime}(-)$ & $0.083(0.005)$ & $\mathbf{0 . 0 5 6 * ( \mathbf { 0 . 0 1 3 } )}$ & $0.078(0.004)$ & $0.091(0.008)$ \\
$\tau_{0}(\mathrm{~Pa} . \mathrm{s})$ & $115.36(14.24)$ & $\mathbf{1 5 6 . 0 2 * ( 1 5 . 7 4 )}$ & $112.15(16.98)$ & $91.23(21.14)$ \\
$\gamma_{\max }(-)$ & $1.84(0.19)$ & $\mathbf{1 . 0 1 * ( \mathbf { 0 . 2 0 } )}$ & $1.97(0.44)$ & $1.60(0.12)$ \\
\hline
\end{tabular}

Fibrin clots containing LMWH or pentasaccharide (S5) at concentrations in the therapeutic range were examined as illustrated in Figs. 3 and 4. The plateau values of the storage modulus $\left(G^{\prime}\right)$, loss modulus $\left(G^{\prime \prime}\right)$ and loss tangent $\left(G^{\prime}, G^{\prime}\right)$ at the end of the clotting phase and the critical shear stress $\left(\tau_{0}\right)$ at the maximal bearable relative deformation $\left(\gamma_{\max }\right)$ before the gel/fluid transition in the fibrin structure are presented as mean (standard deviation). Asterisks indicate $p<0.05$ according to Kolmogorov-Smirnov test in comparison to pure fibrin, $\mathrm{n}=4-6$.

\subsection{Lytic susceptibility of fibrin formed in the presence of polyanions}

In addition to the increased mechanical stability, most polyanions rendered clots resistant to lysis, too. All polyphosphates, regardless of their size, prolonged plasmin-mediated fibrinolysis (Fig. 5). Despite their effects on clot formation and structure, most heparins did not alter the lysability of preformed composite clots by surface-applied plasmin (Fig. 6). Interestingly, the fully desulfated UFH variant and the pentasaccharide shortened lysis-times (Fig. 6). In the intrinsic lysis setup, fibrin dissolution with incorporated plasmin was hardly affected by the applied heparin derivatives. Blocking the high-affinity lysine-binding sites of plasmin with $0.5 \mathrm{mM} 6$-aminohexanoate not only abolished the effects of the polyanions, if 
they were inhibitory (polyphosphates), but lysis with kringle-blocked plasmin was even accelerated in the polyanion-modified fibrin structure, if the polyanion was not inhibitory on its own (Table 6, heparin derivatives in extrinsic lysis).
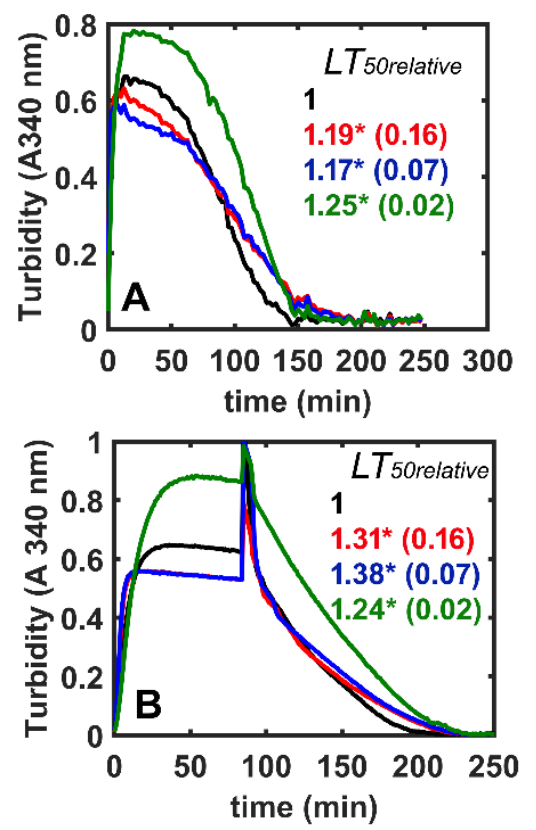

Fig. 5 Polyphosphates inhibit the lysis of fibrin by plasmin. (A) Intrinsic lysis: Clots were prepared from 4.5 $\mu \mathrm{M}$ fibrinogen containing no additive (black) or polyphosphates of 45 (red), 100 (blue) or 700 (green) units at monomeric concentration of $400 \mu \mathrm{M}, 6 \mathrm{nM}$ plasmin and $20 \mathrm{nM}$ thrombin for intrinsic lysis with homogenously dispersed plasmin. (B) Extrinsic lysis: Clots with the same composition as in Panel A were prepared, but without plasmin, and following complete clotting fibrinolysis was initiated with $500 \mathrm{nM}$ plasmin layered on the clot surface. Formation and lysis of clots was monitored by continuous recording of the absorbance at $340 \mathrm{~nm}\left(A_{340 \mathrm{~nm}}\right)$. Curves represent the mean of 5 experimental traces taken in the same microplate for each clot-type and lysis times $\left(L T_{50}\right)$ defined as the time needed to decrease clot turbidity to its half-maximal value are indicated as mean (standard deviation) in relative units compared to pure fibrin using the same color-coding. Asterisks indicate $p<0.05$ according to Kolmogorov-Smirnov test in comparison to pure fibrin, $\mathrm{n}=15$ measured on different days.
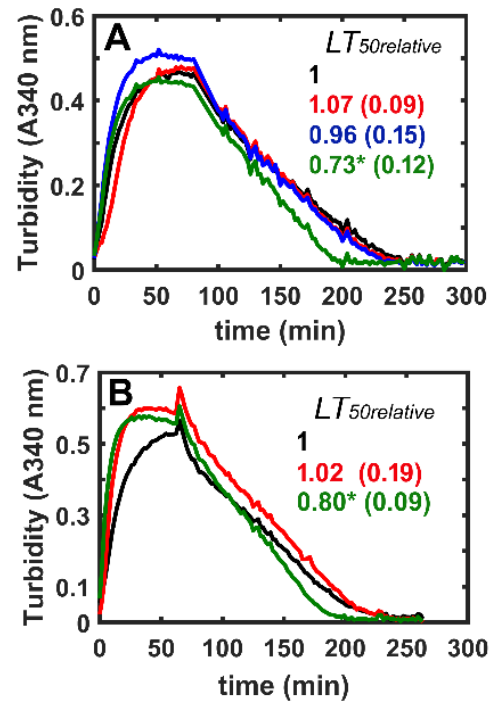

Fig. 6 Extrinsic fibrinolysis: modulation of fibrin formation and lysis by heparin derivatives of various size and charge. (A) Clots were prepared from $4.5 \mu \mathrm{M}$ fibrinogen containing no additive (black) or parent (red), partially (blue), or fully (green) desulfated unfractionated heparin at $2.5 \mathrm{mg} / \mathrm{l}$ by mixing with 8 $\mathrm{nM}$ thrombin. Fibrinolysis was initiated by $500 \mathrm{nM}$ plasmin layered on the clot surface after complete clotting. (B) Composite fibrin/low molecular-weight heparin clots were pre-formed and lysed as in panel A, using the following additives: none (black), LMWH (red) and pentasaccharide (green) at $2.5 \mathrm{mg} / 1$. Formation and lysis of clots was monitored by continuous recording of the absorbance at $340 \mathrm{~nm}\left(A_{340 \mathrm{~nm}}\right)$. Curves represent the mean of 5 experimental traces taken in the same microplate for each clot-type and lysis times $\left(L T_{50}\right)$ are indicated as mean (standard deviation) in relative units compared to pure fibrin using the same color-coding. Asterisks indicate $p<0.05$ according to Kolmogorov-Smirnov test in comparison to pure fibrin, $n=15$ measured on different days. 
Table 6. Blockage of high-affinity lysine-binding sites of plasmin antagonizes the inhibition of fibrinolysis by polyanions.

\begin{tabular}{lccccccc}
\hline Additive & none & UFH & LMWH & S5 & P45 & P100 & P700 \\
\hline Extrinsic lysis setup & 1 & $\mathbf{0 . 7 8 *}$ & $\mathbf{0 . 7 6}^{*}$ & 0.93 & $\mathbf{0 . 8 6}^{*}$ & $\mathbf{0 . 7 4 *}$ & $\mathbf{0 . 7 3 *}$ \\
& $(0.16)$ & $\mathbf{( 0 . 1 7 )}$ & $\mathbf{( 0 . 1 9 )}$ & $(0.14)$ & $\mathbf{( 0 . 1 0 )}$ & $\mathbf{( 0 . 1 2 )}$ & $\mathbf{( 0 . 1 3 )}$ \\
Intrinsic lysis setup & 1 & 0.99 & 1.04 & 1.11 & $\mathbf{0 . 7 7 *}$ & $\mathbf{0 . 8 0 *}$ & $\mathbf{0 . 7 8 *}$ \\
& $(0.10)$ & $(0.06)$ & $(0.27)$ & $(0.17)$ & $\mathbf{( 0 . 1 5 )}$ & $\mathbf{( 0 . 1 4 )}$ & $(\mathbf{0 . 1 5})$ \\
\hline
\end{tabular}

460

461

462

463

464

465

466

467

6-Aminohexanoate $(0.5 \mathrm{mM})$ was added to plasmin prior to its application in the same extrinsic and intrinsic fibrinolytic setups as presented in Figs. 5 and 6. Turbidimetric lysis times $\left(L T_{50}\right)$ were computed in all experimental series: the lysis of pre-formed clots containing $2.5 \mathrm{mg} / \mathrm{l}$ heparin-variant or $400 \mu \mathrm{M}$ polyphosphate, as well as fibrinolysis with incorporated 6AH-blocked plasmin in the presence of the same additives. Lysis times are presented as mean (standard deviation) of the ratio of $L T_{50}$ values of fibrin containing the respective additive and pure fibrin clots with $6 \mathrm{AH}-$ blocked plasmin. Asterisks indicate $p<0.05$ according to Kolmogorov-Smirnov test in comparison to pure fibrin, $\mathrm{n}=5-15$.

\section{Discussion}

For a long time, the biological significance of the formation and dissolution of fibrin had been focused primarily to the cessation of bleeding and the building or removal of intravascular thrombi. The recent accumulation of experimental data on the details of the wound healing process, as well as the formation of extravascular fibrin related to inflammation and tumor development have widened the area of blood coagulation/fibrinolysis research and helped to understand the pieces of information observed earlier (Laurens et al., 2006; Brown and Barker, 2014; Martinod and Wagner, 2014; Varju et al., 2015; Engelmann and Massberg, 2013; Loof et al., 2014; Nickel et al., 2015).

Sulfation pattern and length are two major structural-functional determinants of heparins not only in their anticoagulant actions, but also in their interactions with other proteins, such as histones, fibrinogen, von Willebarnd factor and growth factors (Hirsh and Fuster, 1994, Paolucci et al., 2002; Young et al., 1993 ). Fibrin clots in the presence of the fully sulfated UFH showed a delayed clotting in the kinetic experiments, the thickest and most heterogenous fibers enclosing the biggest pores by SEM and fluid permeability measurements, and mechanically a more viscous gel, which can tolerate a higher degree of deformation. Since our purified system was devoid of antithrombin, these mostly sulfation-dependent effects could have been exerted by the lower thrombin activity in the ternary thrombin-fibrin-heparin complex, as observed earlier (Hogg et al, 1996), and/or by an interference with the molecular steps of fibrin polymerization. The latter possibility is also supported by our earlier observation on the perturbed longitudinal assembly of fibrin monomers in the presence of heparin (Longstaff et al., 2013). In contrast, the shorter heparins accelerated fibrin network assembly, and a denser, more uniform structure was formed, supported by our previous structural data for a smaller periodically repeating unit cell size in the LMWH-modified network (Longstaff et al., 2016). We found the shortest clotting time with S5, whereas the lowest fluid permeability with LMWH. LMWH-modified fibrin clots are more rigid, less viscous, and tolerate a smaller deformation, suggesting that the incorporation of LMWH into the clot restricts intrafibrillar rearrangements upon mechanical deformation (Ryan et al., 1999). Our present data support and further extend earlier observations on the partially opposing effects of UFH and LMWHs on fibrin structure (Collen et al., 2000). Previous studies showed that neither the short chondroitin sulfate/dermatan sulfate or the large, but non-sulfated hyaluronic acid strengthened the clot 
structure (Rottenberg et al., 2016, Komorowicz et al., 2017). The shortest heparin-variant, S5, could not strengthen clot structure, either, maybe because it is too short to carry sufficient electric charge.

Interestingly, we found several equivocal size-dependent effects with another linear polyanion of completely different chemical structure, polyphosphates. Similarly to UHF, the P700-modified clots presented with delayed clotting times, thicker fibers and the decreased overall fluid permeability might be attributed to a rather inhomogeneous network structure. Such clots could tolerate a higher degree of deformation before mechanical disintegration (Tables 2, 4). The shorter polyphosphates, with size corresponding to the platelet-derived types, accelerated fibrin formation, resulted in thicker fibrin fibers and such composite clots showed rheological properties very similar to clots with LMWH: faster time-course of strengthening, less viscous, stronger network structure, and mechanical failure at a higher critical stress due to a stiffer structure and a smaller degree of maximal deformation.

Literature data on the modulation of clot structure by polyphosphates are inconsistent and controversial depending on the experimental setup. Plasma clot structure is highly dependent on the rate of thrombin generation, which in turn is polyphosphate size-dependent: $\approx 100$ monomers are optimal for clotting, if it is initiated through the tissue factor/factor Xa pathway, whereas $\geq 250$ monomers are efficient accelerators of factor XII activation, and hence, thrombin generation (Smith et al., 2010). Data gained in purified systems looked at the shorter, platelet-type polyphosphate size ( $\leq 100$ monomers), and are sometimes conflicting, probably depending on the applied fibrinogen and thrombin concentrations (Smith and Morrissey, 2008; Mutch et al., 2010; Whyte et al., 2016). Our current data suggest clear size-dependent differences in the effects of the large polyanions (UFH, P700) versus the shorter ones (LMWH, P100, P45), and a charge-dependency for UFH-effects.

Fibrinolytic resilience of the polyanion/fibrin clots was the other focus of our current studies. Polyphosphates have been found to prolong tPA-mediated fibrinolysis, and the prolongation was attributed to a decreased binding of both plasminogen and tPA to fibrin, which hampered its cofactor function in the plasminogen activation by tPA (Smith and Morrissey, 2008). In our study, polyphosphates of all sizes prolonged fibrinolysis with surface-applied plasmin, independently of the size-dependent changes in fibrin network architecture. Previous observations with a platelet-type polyphosphate of 75 monomers are equivocal with our model, where fibrinolysis with incorporated plasmin was also retarded. In our current work this dissolution-inhibitory effect was just mildly size-dependent, with P700 and P45/P100 causing ca $25 \%$ and $18 \%$ prolongation, respectively. Plasmin(ogen) relies on its Lys-binding sites located on kringle domains for fibrin binding and here we observed that plasmin with 6aminohexanoate-blocked high affinity Lys-binding sites could solubilize the polyphosphate/fibrin clots not slower, but even faster than the pure fibrin clots- The potential modulatory effect of heparins on the rate of fibrin dissolution was studied and debated a long time ago. Such observations were biased by various evaluation methods for turbidimetric experiments, and the plasma environment, where the antithrombin-heparin binding removed a significant portion of thrombin in the clot formation phase. In intrinsic fibrinolytic models this lower thrombin activity might have lead to incomplete clotting appearing as a shortened dissolution. Similarly to the polyphosphate effects discussed above, 6-aminohexanoate-blocked plasmin could lyse fibrin clots containing UFH variants faster than pure fibrin clots, and at about the same rate observed with native plasmin in fibrin containing fully desulfated UFH. We have recently described a similar, kringle-dependent inhibition of plasmin-mediated fibrinolysis by hyaluronic acid, another polyanion (Komorowicz et al., 2017). Interestingly, the S5-modified clots were lysed faster with native plasmin than the pure fibrin, and this faster rate 
was not further modified by 6-aminohexanoate. This observation suggests that some plasminbinding sites, which hamper fast lysis would be unavailable in the S5-modified clot structure.

\section{Conclusion}

We have found size-, and charge-dependent modulation of the formation, structure, and mechanical properties of fibrin by two biologically relevant polyanion-series, heparins and polyphosphates. Despite the differentially altered structural properties, polyphosphates of all sizes result in a kringle-dependent inhibition of plasmin-mediated fibrinolysis, whereas heparins are minor fibrinolytic modulators. Our data could serve as a biochemical background for the further development of fibrin modulators and fibrinolytic modulators, as well as could give hints for the better understanding of pathophysiological processes after a careful consideration of the natural sources of each polyanion: the large UFH and polyphosphates could naturally occur at sites of microbial entry and inflammation, whereas shorter polyphosphates could be released by activated platelets.

\section{Acknowledgments}

This work was supported by the Hungarian National Research, Development and Innovation Office (NKFIH) (129528, KK) and the Higher Education Institutional Excellence Programme of the Ministry of Human Capacities in Hungary for the Molecular Biology thematic programme of Semmelweis University (KK). The authors are grateful to John Hogwood and Elaine Gray for providing the heparin derivatives and to Györgyi Oravecz and Krisztián Bálint for technical assistance.

\section{Conflict of interest}

The authors declare that the research was conducted in the absence of any commercial or financial relationships that could be construed as a potential conflict of interest.

\section{References}

Brown, A.C., and Barker, T.H. (2014). Fibrin-based biomaterials: Modulation of macroscopic properties through rational design at the molecular level. Acta Biomaterials. 10, 1502-1514. DOI: 10.1016/j.actbio.2013.09.008

Collen, A., Smorenburg, S.M., Peters, E., Lupu, F., Koolwijk, P., Van Norden, C., et al. (2000). Unfractionated and low molecular heparin affect fibrin structure and angiogenesis in vitro. Cancer Res. 60, 6196-6200.

Engelmann, B., and Massberg, S. (2013). Thrombosis as an intravascular effector of innate immunity. Nat. Rev. Immunol. 13, 34-45. DOI: 10.1038/nri3345

Fryer, A., Huang, Y.C., Rao, G., Jacoby, D., Mancilla, E., Whorton, R., et al. (1997). Selective O-desulfation produces nonanticoagulant heparin that retains pharmacological activity in the lung. J. Pharmacol. Exp. Ther. 282, 208-219. 


\section{Heparins and polyphosphates in fibrin}

Hirsh, J., and Fuster, V. (1994). Guide to anticoagulant therapy, Part I. Heparin. Circulation. 89, 1449-1468.

Hogg, P.J., Jackson, C.M., Labanowski, J.K., and Bock, P.E. (1996). Binding of fibrin monomer and heparin to thrombin in a ternary complex alters the environment of the thrombin catalytic site, reduces affinity for hirudin, and inhibits cleavage of fibrinogen. J. Biol. Chem. 271, 26088-26095. DOI: 10.1074/jbc.271.42.26088

Janmey, P.A., Winer, J.P., and Weisel, J.W. (2009). Fibrin gels and their clinical and bioengineering applications. J. R. Soc. Interface. 6, 1-10. DOI: 10.1098/rsif.2008.0327

Jouni, R., Zöllner, H., Khadour, A., Wesche, J., Grotevendt, A., Brandt, S., et al. (2016). Partially desulfated heparin modulates the interaction between antiprotamine/heparin antibodies and platelets. Thromb. Haemost. 115, 324-332. DOI: 10.1160/TH15-07-0539

Komorowicz, E., Balázs, N., Varga, Z., Szabó, L., Bóta, A., and Kolev, K. (2017). Hyaluronic acid decreases the mechanical stability, but increases the lytic resistance of fibrin matrices. Matrix Biol. 63, 55-68. DOI: 10.1016/j.matbio.2016.12.008

Laurens, N., Koolwijk, P., and De Maat, M.P.M. (2006). Fibrin structure and wound healing. J. Thromb. Haemost. 4, 932-939. DOI: 10.1111/j.1538-7836.2006.01861.x

Longstaff C. (2018). Measuring fibrinolysis: from research to routine diagnostic assays. J. Thromb. Haemost. 16, 652-662. DOI: 10.1111/jth.13957

Longstaff, C., Hogwood, J., Gray, E., Komorowicz, E., Varjú, I., Varga, Z., et al. (2016). Neutralisation of the anti-coagulant effects of heparin by histones in blood plasma and purified systems. Thromb. Haemost. 115, 591-599. DOI: 10.1160/TH15-03-0214

Longstaff, C., Thelwell, C., Williams, S.C., Silva, M.M., Szabó, L., and Kolev, K. (2011). The interplay between tissue plasminogen activator domains and fibrin structures in the regulation of fibrinolysis: kinetic and microscopic studies. Blood 117, 661-668. doi: 10.1182/blood-2010-06-290338

Longstaff, C., Varjú, I., Sótonyi, P., Szabó, L., Krumrey, M., Hoell, A., et al. (2013). Mechanical stability and fibrinolytic resistance of clots containing fibrin, DNA and histones. J. Biol. Chem. 288, 6946-6956. DOI: 10.1074/jbc.M112.404301

Loof, T.G., Deicke, C., and Medina, E. (2014). The role of coagulation/fibrinolysis during Streptococcus pyogenes infection. Front. Cell. Inf. Microbiol. 4, 128. DOI: 10.3389/fcimb.2014.00128

Lundblad, R.L., Kingdon, H.S., and Mann, K.G. (1976). Thrombin. Methods Enzymol. 45, 156-176.

Martinod, K., and Wagner, D.D. (2014). Thrombosis: tangled up in NETs. Blood. 123, 2768-2776. DOI: 10.1182/blood-2013-10-463646

Morrissey, J.H., Choi, S.H., and Smith, S.A. (2012). Polyphosphate: an ancient molecule that links platelets, coagulation, and inflammation. Blood. 119, 5972-5979. DOI: 10.1182/blood-2012-03-306605 
Mossesson, M.W. (2005). Fibrinogen and fibrin structure and functions. J. Thromb. Haemost. 3, 1894-1904. DOI: 10.1111/j.1538-7836.2005.01365.x

Mullarky, I.K., Szaba, F.M., Berggren, K.N., Parent, M.A., Kummer, L.W., Chen, W., et al. Infection-stimulated fibrin deposition controls hemorrhage and limits hepatic bacterial growth during Listeriosis. (2005). Infect. Immun. 73, 3888-3895. DOI: 10.1128/IAI.73.7.3888-3895.2005

Mutch, N.J., Engel, R., de Willige, S.U., Philippou, H., and Ariens, R.A.S. (2010). Polyphosphate modifies the fibrin network and down-regulates fibrinolysis by attenuating binding of tPA and plasminogen to fibrin. Blood. 115, 3980-3988. DOI: 10.1182/blood2009-11-254029

Nagasawa, K., Inoue, Y., and Kamata, T. (1977). Solvolytic desulfation of glycosaminoglycuronan sulfates with dimethyl sulfoxide containing water or methanol. Carbohydr. Res. 58, 47-55.

Nenci, G.G., Parise, P., Morini, M., Rossini, A., and Agnelli, G. (1992). Fibrin clot obtained from plasma containing heparin show a higher sensitivity to t-PA-induced lysis. Blood Coagul. Fibrinolysis. 3, 279-285.

Nickel, K.F., Ronquist, G., Langer, F., Labberton, L., Fuchs, T.A., Bokemeyer, C., et al. (2015). The polyphosphate-factor XII coagulation drives coagulation in prostate cancerassociated thrombosis. Blood. 126, 1379-1389. DOI: 10.1182/blood-2015-01-622811

Nikolova, N.D., Toneva-Zheynova, D., Kolev, K., and Tenekedjiev, K. (2013). "Monte Carlo statistical tests for identity of theoretical and empirical distributions of experimental data." In Theory and Applications of Monte Carlo Simulations, ed. W.K. Chan (London, UK: InTech), 1-26. DOI: 10.5772/53049

Paolucci, F., Claviés, M.C., Donat, F., and Necciari, J. (2002). Fondaparinux Sodium Mechanism of Action. Identification of Specific Binding to Purified and Human PlasmaDerived Proteins. Clin. Pharmacokinet. 41(Suppl. 2), 11-18. DOI: 10.2165/00003088200241002-00002

Parise, P., Morini, M., Agnelli, G., Ascani, A., and Nenci, G.G. (1993). Effects of low molecular weight heparins on fibrin polymerization and clot sensitivity to rPA-induced lysis. Blood Coagul. Fibrinolysis. 4, 721-727.

Rottenberger, Z., Komorowicz, E., Szabó, L., Bóta, A., Varga, Z., Machovich R, et al. (2013). Lytic and mechanical stability of clots composed of fibrin and blood vessel wall components. J. Thromb. Haemost. 11, 529-538. DOI: 10.1111/jth.12112

Ruiz, F.A., Lea, C.R., Oldfield, E., and Docampo, R. (2004). Human platelet dense granules contain polyphosphate and are similar to acidocalcisomes of bacteria and unicellular eukaryotes. J. Biol. Chem. 279, 44250-44257. DOI: 10.1074/jbc.M406261200

Ryan, E.A., Mockros, L.F., Weisel, J.W., and Lorand L. (1999). Structural origins of fibrin clot rheology. Biophys. J. 77, 2813-2826. DOI: 10.1016/S0006-3495(99)77113-4

Semeraro, F., Piro, D., Rossiello, M.R., Ammollo, T., and Colucci, M. (2007). Profibrinolytic activity of the direct thrombin inhibitor melagatranand unfr actionated 
heparin in platelet-poor and platelet-rich clots. Thromb. Haemost. 98, 1208-1214. DOI: 10.1160/TH07-05-0375

Smith, S.A., and Morrissey, J.H. (2008). Polyphosphate enhances fibrin clot structure. Blood. 112, 2810-2816. DOI: 10.1182/blood-2008-03-145755

Smith, S.A., Choi, S.H., Davis-Harrison, R., Huyck, J., Boettcher, J., Rienstra, C.M., et al. (2010). Polyphosphate exerts differential effects on blood clotting, depending on polymer size. Blood. 116, 4353-4359. DOI: 10.1182/blood-2010-01-266791

Travers, R.J., Shenoi, R.A., Kalathottukaren, M.T., Kizhakkedathu, J.N., and Morrissey, J.H. (2014). Nontoxic polyphosphate inhibitors reduce thrombosis while sparing hemostasis. Blood. 124, 3183-3190. DOI: 10.1182/blood-2014-05-577932

Undas, A., and Ariens, R.A.S. (2011). Fibrin clot structure and function. A role in the pathophysiology of arterial and venous thromboembolic diseases. Arterioscler. Thromb. Vasc. Biol. 31, e88-e99. DOI: 10.1161/ATVBAHA.111.230631

Varin, R., Mirshahi, S., Mirshahi, P., Kierzek, G., Sebaoun, D., Mishal, Z., et al. (2007). Clot structure modification by fondaparinux and consequence on fibrinolysis: A new mechanism of antithrombotic activity. Thromb. Haemost. 97, 27-31. DOI:10.1160/TH0607-0394

Varjú, I., Longstaff, C., Szabó, L., Farkas, Á.Z., Varga-Szabó, V.J., Tanka-Salamon, A., et al. (2015). DNA, histones and neutrophil extracellular traps exert anti-fibrinolytic effects in a plasma environment. Thromb. Haemost. 113, 1289-1298. DOI: 10.1160/TH14-08-0669

Wan, J.G., Mu, J.S., Zhu, H.S., and Geng, J.G. (2002). N-desulfated non-anticoagulant heparin inhibits leukocyte adhesion and transmigration in vitro and attenuates acute peritonitis and ischemia and reperfusion injury in vivo. Inflamm. Res. 51, 435-443

Weisel, J.W., and Litvinov, R.I. (2008). The biochemical and physical process of fibrinolysis and effects of clot structure and stability on the lysis rate. Cardiovasc. Hematol. Agents Med. Chem. 6, 161-180. DOI : 10.2174/187152508784871963

Weisel, J.W., and Litvinov, R.I. (2013). Mechanisms of fibrin polymerization and clinical implications. Blood. 121: 1712-1719. DOI: 10.1182/blood-2012-09-306639

Weitz, J., Kuint, J., Leslie, B., and Hirsh, J. (1991). Standard and low molecular weight heparin have no effect on tissue plasminogen activator induced plasma clot lysis or fibrinogenolysis. Thromb. Haemost. 65, 541-544. DOI: 10.1055/s-0038-1648186

Whyte, C.S., Chernysh, I.N., Domingues, M.M., Connell, S., Weisel, J.W., Ariens, R.A.S., et al. (2016). Polyphosphate delays fibrin polymerisation and alters the mechanical properties of the fibrin network. Thromb. Haemost. 116, 897-903. DOI: 10.1160/TH16-01-0062

Young, E., Cosmi, B., Weitz, J., and Hirsh, J. (1993). Comparison of the non-specific binding of unfractionated heparin and low molecular weight heparin (enoxaparin) to plasma proteins. Thromb. Haemost. 70, 625-630. DOI: 10.1055/s-0038-1649639 


\section{Supplementary Material}

\section{Biorelevant polyanions stabilize fibrin against mechanical and proteolytic decomposition: effects of polymer size and electric charge}

\section{Erzsébet Komorowicz ${ }^{1}$, Nóra Balázs ${ }^{1}$, Anna Tanka-Salamon', Zoltán Varga ${ }^{2}$, László Szabó $^{1}$, Attila Bóta ${ }^{2}$, Colin Longstaff ${ }^{3}$, Krasimir Kolev ${ }^{*}$}

${ }^{1}$ Department of Medical Biochemistry, Semmelweis University, Budapest, Hungary

${ }^{2}$ Department of Biological Nanochemsitry, Institute of Materials and Environmental Chemistry, Research Centre for Natural Sciences, Hungarian Academy of Sciences, Budapest, Hungary

${ }^{3}$ Biotherapeutics, Haemostasis Section, National Institute for Biological Standards and

Control, South Mimms, Potters Bar, UK

When making rheological measurements in an oscillatory rheometer there is always a possibility for the "wall slip" phenomenon to occur. During wall slip the cohesive forces within the studied material overcome the cohesive forces between the material and the metal plate/cone parts of the rheometer, leading to improper and uneven force transmission to the sample. This could result in strange curve shapes with dog-legs, elbows, bad reproducibility, or overestimation of the force required to yield the desired deformation. Some authors have studied the factors which could minimize the probability of a wall slip, while others aimed to quantitate it (Walter et al., 2017; Bertula et al., 2019).

According to data in the literature there are several factors, which can minimize the probability of wall slip, and which apply also in our specific experimental setup:

1) In our settings clots are formed in situ in the gap space between the horizontal plate and the measuring cone head, since the cone head is lowered into gap position 20 seconds after the addition of thrombin, when the sample is still fluid enough so that at the arrival of the cone (2 degrees angle, $35 \mathrm{~mm}$ diameter) into the 105 um gap position it can evenly distribute the sample in the gap space. If the clot is already firm, the arriving cone head pushes it to the side, since the clot otherwise would be of smaller diameter, but higher than the gap space. Earlier studies have found that polymerizing samples in situ in the instrument greatly enhances its attachment to the plates, while putting ex situ polymerized preformed samples into the gap space of parallel-plate rheometers results in poor attachment and favors wall slip (Bertula et al., 2019). In our experiments we allow the clot to be formed in the gap for 2 minutes and we start with the oscillatory deformation cycles only after this 2-min interval to collect the data for the kinetic curves of clot strengthening.

2) We and other authors (Walter et al., 2017) have visually observed that after the measurements both cone and plate have to be thoroughly cleaned because of firmly attached clot remnants. In our hands the clots had to be wiped off the surfaces, and the metal surfaces cleaned, which also suggests a strong cohesion between the clot and the instrument.

3) Other authors (Bertula et al., 2019) who studied the wall slip phenomenon in details reasoned and found that without wall slip the storage and loss moduli, as characteristic viscoelastic parameters for the material itself, should be independent on the gap size, i.e. the sample thickness. If wall slip occurs, these parameters should change with the gap size, since a wall slip would add improper force transition to the sample resulting in an overestimation of the force requirements. Also, in shear stress controlled experiments the slip velocity would 
depend only on the applied shear stress, and would add a gap height-dependent factor to the true shear rate resulting in an apparent shear rate which would turn then gap size-dependent. As a result, elastic moduli and viscosity would also turn gap size-dependent instead of being a parameter characterizing the material itself.

We tested therefore the influence of gap size on the viscoelastic parameters of pure fibrin determined in our instrument with our usual settings, as described in Materials and Methods. We present 5-5 original tracings of storage and loss moduli in the course of clot formation, which show good reproducibility and good concordance at the 3 different gap sizes tested (Figure S1).

In Table S1 numerical parameters computed from kinetic and stress-controlled flow-type experiments, as detailed in Materials and Methods are collected including statistical analysis. We found that raising the gap size from 105 to $150 \mu \mathrm{m}$ did not cause any change in any of the 5 parameters, and at $200 \mu \mathrm{m}$ gap size the only parameter which started to increase was the loss tangent reflecting the relative energy loss during deformation. The kinetic curves showing storage and loss moduli in the course of the clotting process showed good reproducibility, and there were no spikes or other abnormalities in the flow curves, which would indicate a wall slip during the experiment. Considering the geometry of our cone head -2 degrees angle and $35 \mathrm{~mm}$ diameter with a default $105 \mu \mathrm{m}$ gap size - we think that with the default gap size the cone head is deeply embedded into the polymerizing sample with the $105 \mu \mathrm{m}$ gap at the center and a $611 \mu \mathrm{m}$ high clot around the rim of the cone. By increasing the gap size up to $200 \mu \mathrm{m}$ we decrease the relative depth of cone incorporation into the sample, which could increase the probability of wall slip. Consequently, we performed all of the reported measurements at a gap of $105 \mu \mathrm{m}$, significantly lower than the gap size $(150 \mu \mathrm{m})$ for which we have the evidence for no wall slip.

Summarizing our experience, specific measurements related to gap size, as well as literature data we could conclude that the probability of wall slip in our standard settings is very low, which we attribute to the carefully selected polymerization conditions (in situ fibrin polymerization) and rheometer geometry (cone-and-plate).

\section{References}

Bertula, K., Martikainen, L., Munne, P., Hietala, S., Klefström, J., Ikkala, O., at al. StrainStiffening of Agarose Gels. (2019). ACS Macro Letters. 8 (6), 670-675 DOI: 10.1021/acsmacrolett.9b00258

Walter, B.L., Pelteret, J.P., Kascht, J., Schubert, D.W., and Steinmann P. (2017). On the wall slip phenomenon of elastomers in oscillatory shear measurements using parallel-plate rotational rheometry: I. Detecting wall slip. Polymer Test. 61, 430-440. DOI: 10.1016/j.polymertesting.2017.05.035 
Table S1. Viscoelastic parameters of fibrin clots measured at various gap-sizes in a coneand-plate oscillatory rheometer. Pure fibrin clots were formed in situ in a cone-and-plate oscillatory rheometer applying various gap-sizes. The plateau values of the storage modulus $\left(G^{\prime}\right)$, loss modulus $\left(G^{\prime}\right.$ ') and loss tangent (G''/G') at the end of the clotting phase and the critical shear stress $\left(\tau_{0}\right)$ at the maximal bearable relative deformation $\left(\gamma_{\max }\right)$ before the gel/fluid transition in the fibrin structure are presented as mean (standard deviation). Asterisks indicate $p<0.05$ according to Kolmogorov-Smirnov test in comparison to the standard gap-size of 105 $\mu \mathrm{m}, \mathrm{n}=5-6$.

\begin{tabular}{llll} 
& \multicolumn{3}{c}{ Gap size $(\mu \mathrm{m})$} \\
\cline { 2 - 4 } & 105 & 150 & 200 \\
\hline $\mathrm{G}^{\prime}(\mathrm{Pa})$ & $44.25(4.00)$ & $40.20(7.35)$ & $48.35(13.46)$ \\
$\mathrm{G}^{\prime \prime}(\mathrm{Pa})$ & $4.22(0.39)$ & $3.93(0.84)$ & $5.06(1.56)$ \\
$\mathrm{G}^{\prime \prime} / \mathrm{G}^{\prime}(-)$ & $0.095(0.003)$ & $0.097(0.005)$ & $0.103^{*}(0.004)$ \\
$\tau_{0}(\mathrm{~Pa} . \mathrm{s})$ & $167.79(30.92)$ & $169.75(28.76)$ & $179.92(33.55)$ \\
$\gamma_{\max }(-)$ & $2.42(0.41)$ & $2.53(0.28)$ & $2.72(0.42)$ \\
\hline
\end{tabular}

Figure S1. Clot strengthening in the course of fibrin polymerization in situ in a cone-andplate rheometer applying various gap-sizes. Fibrinogen $(7.4 \mu \mathrm{M})$ was mixed with $10 \mathrm{nM}$ thrombin and storage modulus (G', continuous line), as well as loss modulus (G', dashed line) were measured in an oscillation rheometer in the course of fibrin polymerization. 5 original tracings are shown for each applied gap-size: the default $105 \mu \mathrm{m}$ (black), as well as $150 \mu \mathrm{m}$ (red), and $200 \mu \mathrm{m}$ (blue). Numerical data for the maximal G' and G', values are summarized in Table S1.

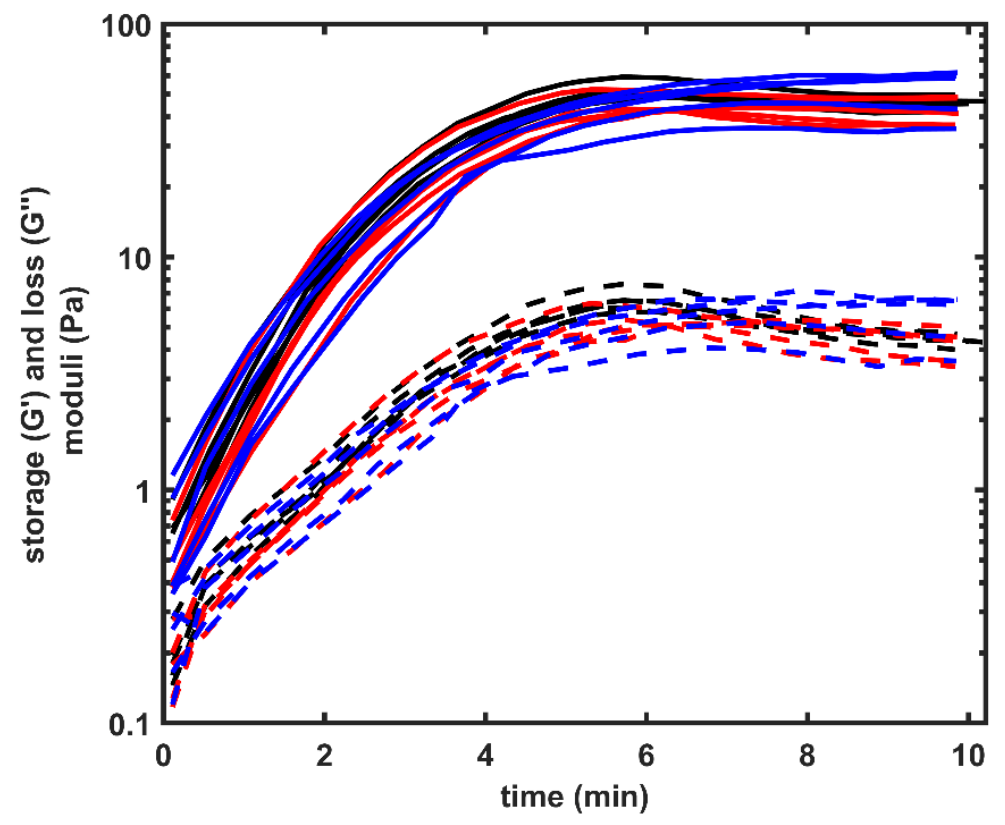

